$\mathrm{DOE} / \mathrm{RW}--0234$

TI89 011304

\title{
Telecommunications Network Plan
}

May 1989

U.S. Department of Energy

Office of Civilian Radioactive Waste Management

Washington, DC 20585

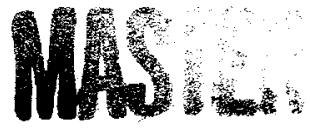




\section{DISCLAIMER}

This report was prepared as an account of work sponsored by an agency of the United States Government. Neither the United States Government nor any agency thereof, nor any of their employees, make any warranty, express or implied, or assumes any legal liability or responsibility for the accuracy, completeness, or usefulness of any information, apparatus, product, or process disclosed, or represents that its use would not infringe privately owned rights. Reference herein to any specific commercial product, process, or service by trade name, trademark, manufacturer, or otherwise does not necessarily constitute or imply its endorsement, recommendation, or favoring by the United States Government or any agency thereof. The views and opinions of authors expressed herein do not necessarily state or reflect those of the United States Government or any agency thereof. 


\section{DISCLAIMER}

Portions of this document may be illegible in electronic image products. Images are produced from the best available original document. 
Section

List of Figures $\ldots \ldots \ldots \ldots \ldots \ldots \ldots \ldots \ldots \ldots \ldots \ldots \ldots \ldots \ldots \ldots \ldots$ iii

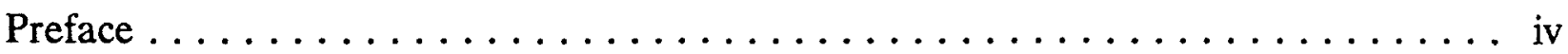

Executive Summary $\ldots \ldots \ldots \ldots \ldots \ldots \ldots \ldots \ldots \ldots \ldots \ldots \ldots$

$1.0 \quad$ INTRODUCTION $\ldots \ldots \ldots \ldots \ldots \ldots \ldots \ldots \ldots \ldots \ldots \ldots$

1.1 PURPOSE OF THE OCRWM TELECOMMUNICATIONS NETWORK PLAN $\ldots \ldots \ldots \ldots \ldots \ldots \ldots \ldots \ldots \ldots \ldots \ldots \ldots \ldots$

1.1.1 Role of the Licensing Support System in Telecommunications

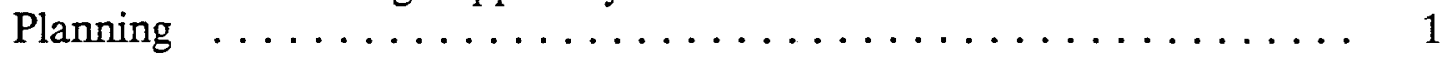

1.2 SCOPE OF THE TELECOMMUNICATIONS NETWORK PLAN . . . 1

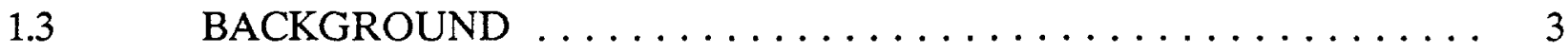

2.0 THE IMPLEMENTATION ENVIRONMENT $\ldots \ldots \ldots \ldots \ldots \ldots$

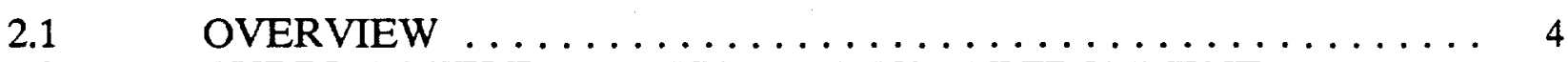

CURRENT TELECOMMUNICATIONS ENVIRONMENT:
SITE PROFILES $\ldots \ldots \ldots \ldots \ldots \ldots \ldots \ldots \ldots \ldots \ldots \ldots \ldots \ldots$

2.2.1 OCRWM Headquarters: Washington D.C. $\ldots \ldots \ldots \ldots \ldots \ldots$

2.2.2 YMPO/SAIC/M\&O: Las Vegas, Nevada .............. 4

2.2.3 RTTD/OWTD: Chicago, Illinois ................ 7

2.2.4 Idaho Falls, Idaho and Oak Ridge, Tennessee $\ldots \ldots \ldots \ldots \ldots \ldots$

$3.0 \quad$ PROPOSED OCRWM TELECOMMUNICATIONS NETWORK . . . . 8

3.1 INTRODUCTION: THE NEED FOR AN INTEGRATED TELECOMMUNICATIONS NETWORK $\ldots \ldots \ldots \ldots \ldots \ldots \ldots, 8$

3.1.1 Problems with the Existing Telecommunications Environment ...... 8

3.1.2 Goals and Objectives of the New OCRWM Telecommunications

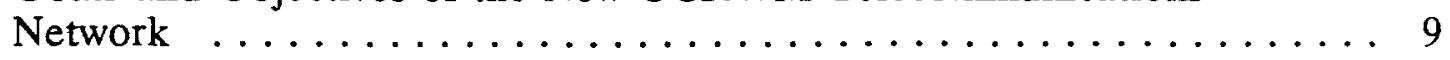

3.2 GENERAL NETWORK REQUIREMENTS AND SPECIFICATIONS . . 9

3.2.1 Conceptual Models and Designs $\ldots \ldots \ldots \ldots \ldots \ldots \ldots \ldots$

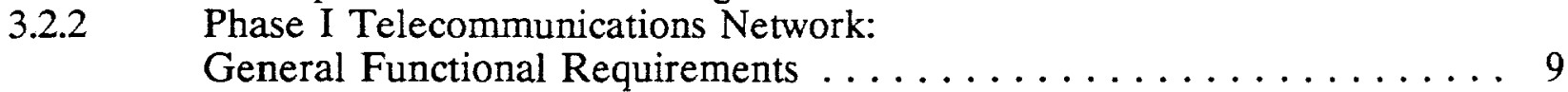


Table of Contents

( Contin u ed)

Section

Page

3.2.3 Operating in a DEC Environment: Node Addresses . . . . . . . . . 10

3.2.4 Network Management, Control, and Maintenance . . . . . . . . . . . . 10

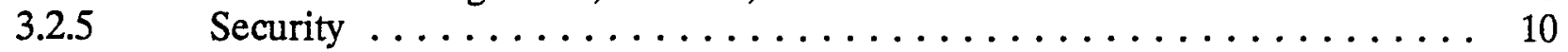

$3.3 \quad$ SITE REQUIREMENTS $\ldots \ldots \ldots \ldots \ldots \ldots \ldots \ldots \ldots \ldots$

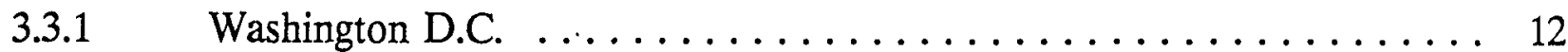

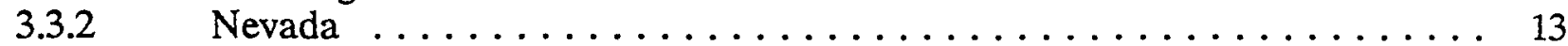

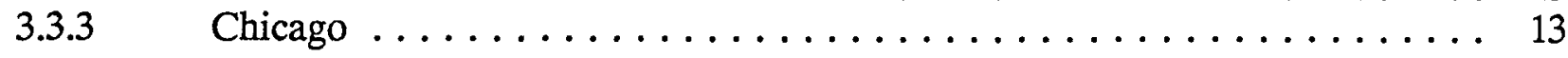

4.0 RELATIONSHIP BETWEEN THE PROPOSED

TELECOMMUNICATIONS NETWORK AND THE

OCRWM LICENSING SUPPORT SYSTEM . . . . . . . . . . . . . . 14

4.1 THE LICENSING SUPPORT SYSTEM:

PURPOSES AND OBJECTIVES .................. 14

4.2 THE RELATIONSHIP BETWEEN THE PROPOSED

TELECOMMUNICATIONS NETWORK AND THE LSS . . . . . . . . 16

4.3 LICENSING SUPPORT SYSTEM TELECOMMUNICATIONS

REQUIREMENTS . . . . . . . . . . . . . . . . . . . . 16

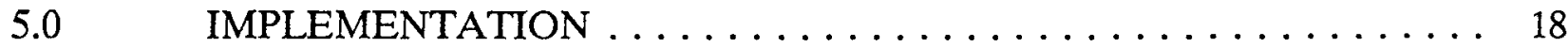

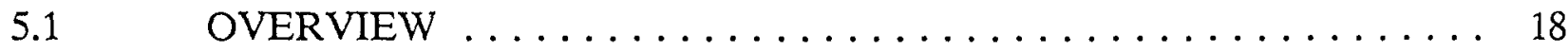

5.1.1 Phase I and Phase II Development and Implementation Schedules . . . 19

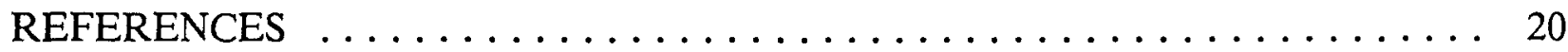

\section{APPENDICES *}
A. Local Area Networks
B. Extended Local Area Networks
C. Remote Extended Local Area Networks
D. Selected Carrier Redundancy Alternatives
E. Bandwidth and Data Line Alternatives

Appendices containing performance specifications will be developed in coordination with CSTM technical support and will be included in future revisions of this plan. 
Section

Page

1. OCRWM WAN - Phase I Geography ................... 2

2. Current OCRWM Communications .................. 5

3. OCRWM Information Systems and Data Bases . . . . . . . . . . . . . 6

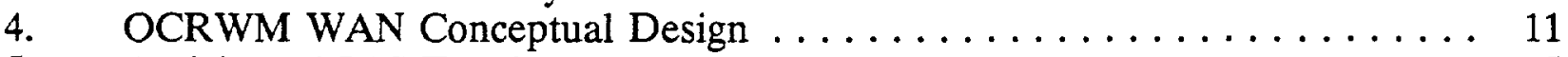

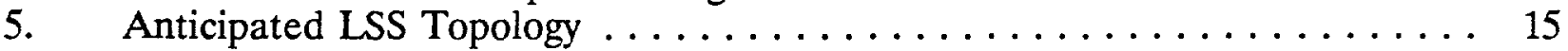

6. Preliminary Design LSS Communications Networks . . . . . . . . . . . . 17 
This plan reflects the Office of Civilian Radioactive Waste Management's (OCRWM) current thinking on the design and development of a program-wide telecommunications network. This plan will be periodically reviewed and revised to ensure that it continues to present a telecommunications strategy fully responsive to the needs of all program participants.

At this time, the OCRWM Licensing Support System (LSS) is still under development. Thus, the requirements set forth herein represent only what is now known about the LSS. OCRWM's Information Resources Management Division (IRMD) will keep abreast of any significant changes in Department of Energy (DOE), OCRWM, and the LSS that would warrant a reorientation of this plan. Should such changes occur, IRMD will revise the plan to accommodate them. In addition, IRMD will work closely with DOE's Office of Computer Services and Telecommunications Management (CSTM) to ensure that the telecommunications goals of OCRWM and DOE are achieved.

With regard to the procurement and installation of telecommunications hardware and software, IRMD will rely on CSTM to develop a technical implementation plan and to assist in preparing performance specifications for all integral components of the planned network (e.g., gateways and bridges, communication service units, communication/data lines). When prepared, these specifications will be included as additional Appendices in subsequent revisions of this plan. 
Executive Sum mary

\section{Need for Information Management}

In order to successfully implement the Nuclear Waste Policy Act (i.e., to site the Nation's first permanent geologic repository for spent fuel and high-level radioactive waste) the Office of Civilian Radioactive Waste Management (OCRWM) must, among other things, be equipped to readily produce, file, store, access, retrieve, and transfer a wide variety of technical and institutional data and information. The data and information regularly produced by members of the OCRWM Program supports, and will continue to support, a wide range of program activities. Some of the more important of these information communication-related activities include:

(1) Supporting the preparation, submittal, and review of a license application to the Nuclear Regulatory Commission (NRC) to authorize the construction of a geologic repository.

(2) Responding to requests for information from parties affected by and/or interested in the program.

(3) Providing evidence of compliance with all relevant Federal, State, local, and Indian Tribe regulations, statutes, and/or treaties.

\section{Purpose of the OCRWM Telecommunications Network Plan}

The OCRWM Telecommunications Network Plan (TNP) is intended to identify, as well as to present the current strategy for satisfying, the telecommunications requirements of the civilian radioactive waste management program. The TNP will set forth the plan for integrating OCRWM's information resources among major program sites. Specifically, this plan will introduce a telecommunications network designed to establish communication linkages across the program's Washington, D.C.; Chicago, Illinois; and Las Vegas, Nevada, sites. The linkages across these and associated sites will comprise Phase I of the proposed OCRWM telecommunications network. The second phase (i.e., Phase II) of OCRWM's effort to develop a program-wide telecommunications network will focus on the modification and expansion of the Phase I network to fully accommodate access to the OCRWM Licensing Support System (LSS). The primary components of the proposed OCRWM telecommunications network include: (a) local area networks; (b) extended local area networks; and (c) remote extended (wide) area networks.

\section{Scope of the OCRWM Telecommunications Network Plan}

The TNP focuses primarily on the near-term (Phase I) wide area telecommunications requirements of the entire civilian radioactive waste management program. The TNP will introduce a multi-phased strategy to facilitate the transfer of information among major 
program sites, and between such sites and selected external parties (e.g., the NRC, the Nuclear Waste Review Board). The driving force behind OCRWM's efforts to develop and implement the TNP is the LSS. Therefore, LSS requirements, to the extent that they are known, will play a prominent role in all aspects of Phase I telecommunications network planning.

\section{Role of the OCRWM Licensing Support System}

As with the OCRWM Office Automation Plan (OAP; January 1989), the primary impetus for developing an OCRWM-wide TNP is the OCRWM Licensing Support System. The LSS is an automated information storage and retrieval system that will assist the Department of Energy (DOE) and the Nuclear Regulatory Commission (NRC) in the licensing of a repository for the disposal of spent nuclear fuel and high-level radioactive waste. It will facilitate the discovery process by containing the relevant licensing information in an electronic data base accessible to interested parties via OCRWM's Phase II telecommunications network. Access to the LSS will be available to interested parties prior to the NRC's consideration of the Department's license application.

\section{Relationship Between the Proposed Telecommunications Network and DOENTS}

In addition to the purposes already stated, implementation of the proposed Phase I and Phase II wide area telecommunications networks is also intended, in part, to support and expand the DOE Nation-wide Telecommunications Services (DOENTS), which already includes INTRANET and the OPMODEL.

\section{Role of CSTM}

As with any effort within DOE to design, develop, and maintain information resources, the Office of Computer Services and Telecommunications Management (CSTM) will play an integral role in ensuring that the desired objectives of such activities are achieved. OCRWM Headquarters will submit the functional requirements for the proposed Phase I and Phase II telecommunications networks detailed in this Plan to CSTM as soon as they are finalized. OCRWM-Headquarters will then work with CSTM to develop the necessary technical implementation plans and procedures for the proposed networks.

\section{Network Implementation}

The development and implementation schedules for the proposed telecommunications network are currently being developed in coordination with CSTM. These schedules will portray a multiphased approach to network development and implementation. The rationale behind "phasing" the introduction of telecommunications technologies into the OCRWM Program is quite simple. A phased approach is "scalable" that is, it affords network designers and implementers the opportunity to adapt to changing functional requirements--to scale the network to the current and anticipated requirements of the network users. Network implementation schedules will be included in subsequent revisions of this plan as they become available. 
The Office of Civilian Radioactive Waste Management (OCRWM) Telecommunications Network Plan (TNP) is intended to identify, as well as to present the current strategy for satisfying, the telecommunications requirements of the civilian radioactive waste management program. The TNP will set forth the plan for integrating OCRWM's information resources among major program sites. Specifically, this plan will introduce a telecommunications network designed to establish communication linkages across the program's Washington, D.C., Chicago, Illinois, and Las Vegas, Nevada sites (see Figure 1). The linkages across these and associated sites will comprise Phase I of the proposed OCRWM telecommunications network. The second phase (i.e., Phase II) of OCRWM's effort to develop a program-wide telecommunications network will focus on the modification and expansion of the Phase I network to fully accommodate access to the OCRWM Licensing Support System (LSS). The primary components of the proposed OCRWM telecommunications network are: (a) local area networks; (b) extended local area networks; and (c) remote extended (wide) area networks. The reader who is not completely familiar with these components should review Appendices A through $\mathrm{E}$ before reading Section 3.0.

\subsubsection{Role of the Licensing Support System in Telecommunications Planning}

As with the OCRWM Office Automation Plan (OAP; November 1988), the primary impetus for developing an OCRWM-wide TNP is the OCRWM Licensing Support System. The LSS is an automated information storage and retrieval system that will assist the Department of Energy (DOE) and the Nuclear Regulatory Commission (NRC) in the licensing of a repository for the disposal of spent nuciear fuel and high-level radioactive waste. It will facilitate the discovery process by containing the relevant licensing information in an electronic data base accessible to interested parties. Access to the LSS will be available to these parties prior to the NRC's consideration of the Department's License Application. The LSS has been designated by the Office of Management and Budget as a "Presidential Priority System."

The TNP focuses primarily on the near-term (Phase I) wide area telecommunications requirements of the entire civilian radioactive waste management program. The TNP will introduce a multi-phased strategy to facilitate the transfer of information among the major program sites identified in Figure 1. OCRWM WAN - Phase 1 Geography, and between such sites and selected external parties (e.g., the NRC, the Nuclear Waste Review Board). As mentioned in the preceding section, the driving force behind the efforts to develop and implement the TNP is the LSS. Therefore, LSS requirements, to the extent that they are known, will play a prominent role in all aspects of Phase I telecommunications network planning. 


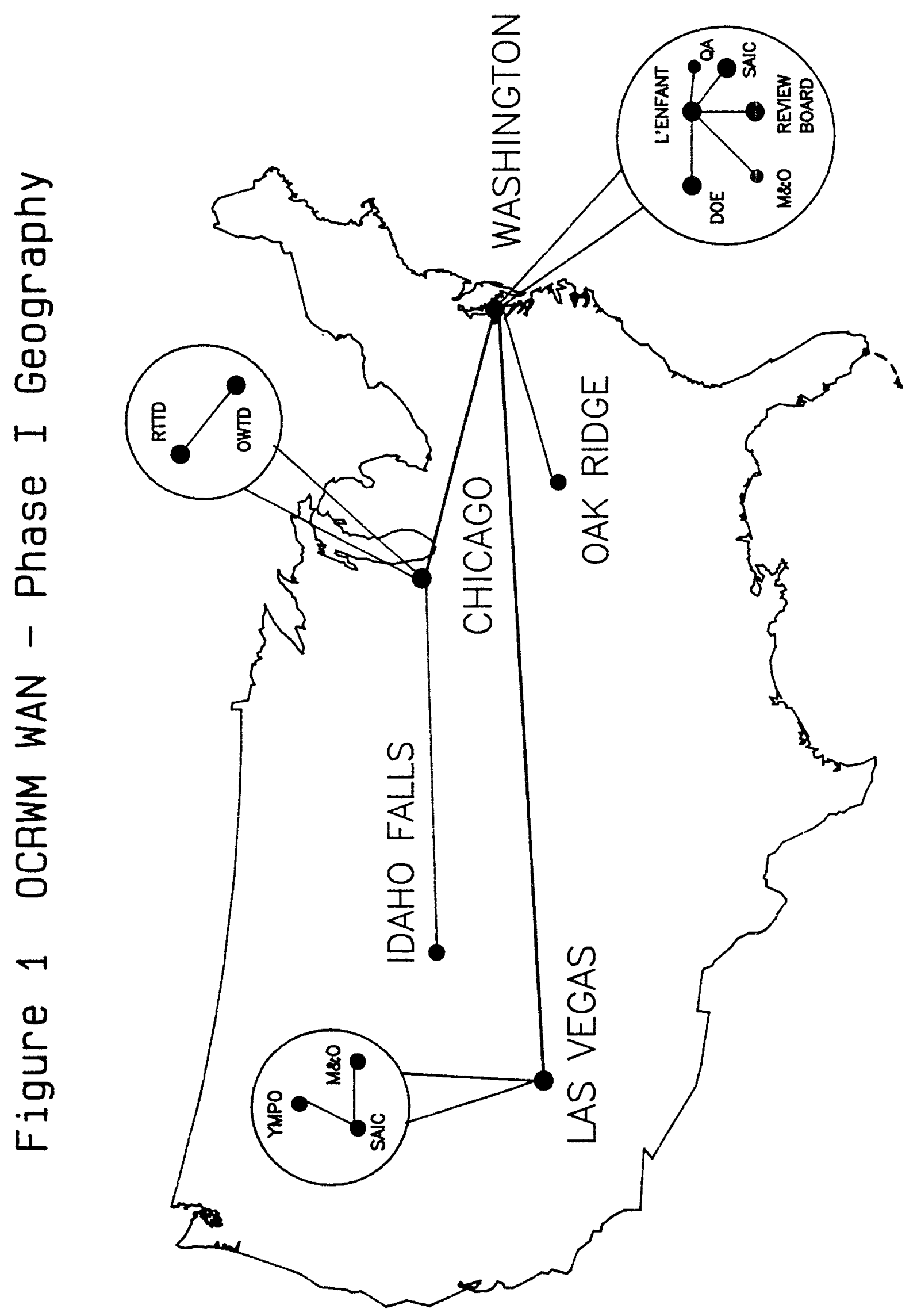


The reader should note that the TNP does not address the topic of intra-site communications (e.g., via local area networks). That topic is the focus of the OCRWM Office Automation Plan (DOE, 1989e). The primary goal here is to identify a strategy for linking the information resources available at specific program sites; and, to make that information accessible to a wider range of parties affected by and/or interested in the OCRWM Program. Given the assumption that potential network sites are, in effect, "islands of information resources," the TNP will present a strategy for ensuring that these islands are linked together so that such resources are available and accessible to a wider audience. Using the analogy of islands of information should help the reader understand the significance and function of the bridges referred to later in the plan.

\subsection{BACKGROUND}

As late as 1986, the OCRWM program had Project Offices in Hanford, Washington (the Basalt Waste Isolation Project--BWIP), Las Vegas, Nevada (the Nevada Nuclear Waste Site Investigation--NNWSI), Chicago, Illinois (the Salt Repository Project Office--SRPO), and Columbus, Ohio (the Crystalline Project Office--CPO). Each Project Office maintained its own information systems, as well as data network. Across Project Offices, these information systems and data networks varied considerably with respect to their technological sophistication. The common trait among them was that none possessed routine processes and/or mechanisms for sharing and transferring information and data resident in their individual systems. This led to a number of problems with the information and data stored across Project Offices.

Central among these was the inability to access information and data resident in another Project Office's system and the consequent duplication of information systems across Project Offices. The fact that data overlapped raised frequent questions as to whose data were correct (i.e., the most up-to-date). The unsettling possibility of receiving a different "answer" to the same question from each Project Office's information system became highly plausible because supposedly identical data bases did not always contain the same data. This problem was most often attributed to differences in maintenance and updating procedures.

The Nuclear Waste Policy Amendments Act of 1987 focused the search for a repository on the Yucca Mountain Site in the State of Nevada. This reorientation of the civilian radioactive waste management program reduced the number of Project Offices participating in the siting of the repository. The remaining Project Offices are located in Chicago, Illinois and Las Vegas, Nevada. These Project Offices, along with OCRWM Headquarters (in Washington, D.C.), form the three primary sites for the Phase I OCRWM telecommunications network. 
Some familiarity with OCRWM's existing telecommunications environment is needed before considering where OCRWM should be headed in terms of telecommunications network design and development. The purpose of this section is to introduce the information resources currently available at each site; that is, the operating hardware that will "figure in" the telecommunications network conceptual design and site-specific requirements for node-to-node communications.

\section{2}

\section{CURRENT TELECOMMUNICATIONS ENVIRONMENT: SITE PROFILES}

In order to familiarize the reader with the current OCRWM telecommunications environment, the following subsections briefly describe the hardware relevant to wide area telecommunications networking at each major Phase I site. These descriptions include information pertaining to the design and development of a telecommunications network fully responsive to both general and specific OCRWM node-to-node communications needs. Figure 1 illustrates Phase I of the proposed OCRWM wide area telecommunications network; Figure 2 depicts the relevant hardware currently operating at the Washington, Chicago, and Las Vegas sites; and, Figure 3 lists OCRWM information systems and data bases that will rely on the telecommunications network.

\subsubsection{OCRWM Headquarters: Washington D.C.}

OCRWM-HQ operates its information systems in a VAX environment. The VAX 8700 , 8300, and MicroVAX IIs (2) are maintained by Roy F. Weston, Incorportated, OCRWMHQ's Technical Support Contractor, to store and process information resident in OCRWM-HQ's major data base and information systems. The Washington M\&O Contractor, QA Contractor, SAIC/McLean, Virginia, and the Nuclear Waste Review Board are not yet part of the D.C. site.

\subsubsection{YMPO/SAIC/M\&O: Las Vegas, Nevada}

A number of VAX components form the foundation for information storage and processing at the Yucca Mountain Project Office (YMPO) and SAIC/Las Vegas. These VAX components include the following: at SAIC - 8700 (2), MicroVAX II (2), 785 (2), and 6240 (1); at YMPO - MicroVAX II (1). The M\&O Contractor is not yet part of the Nevada site. 


\section{Figure 2 Current OCRWM Communications}

LAS VEGAS, NEVADA

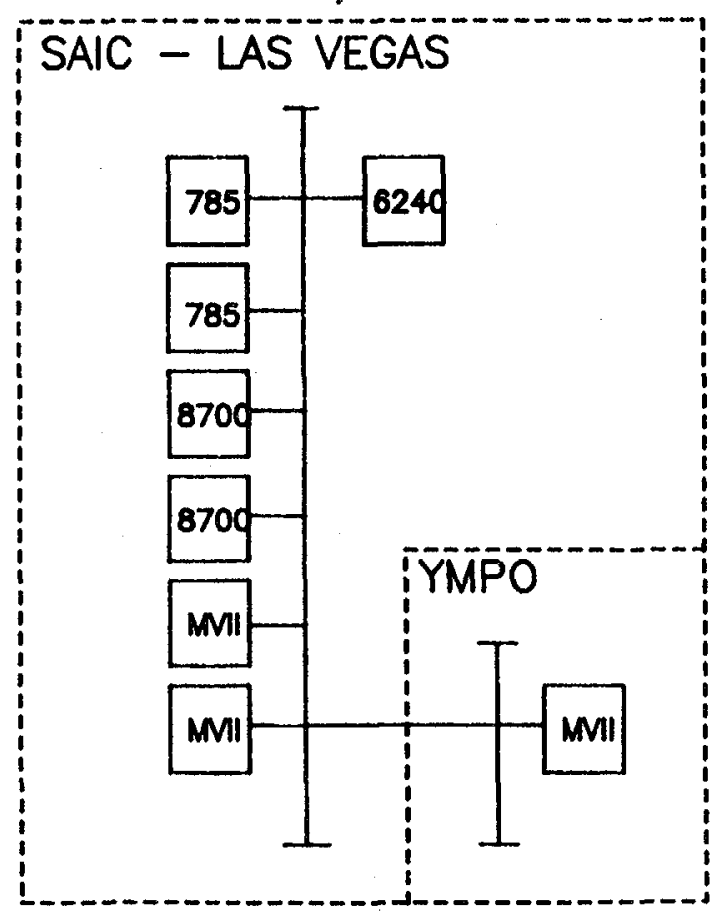

CHICAGO, ILLINOIS

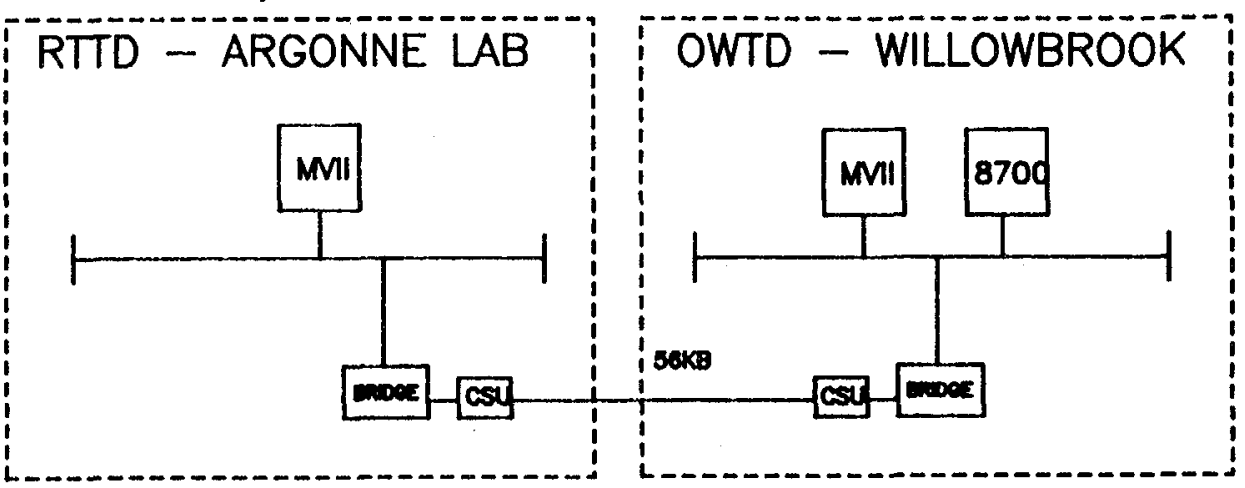

WASHINGTON, D. C.

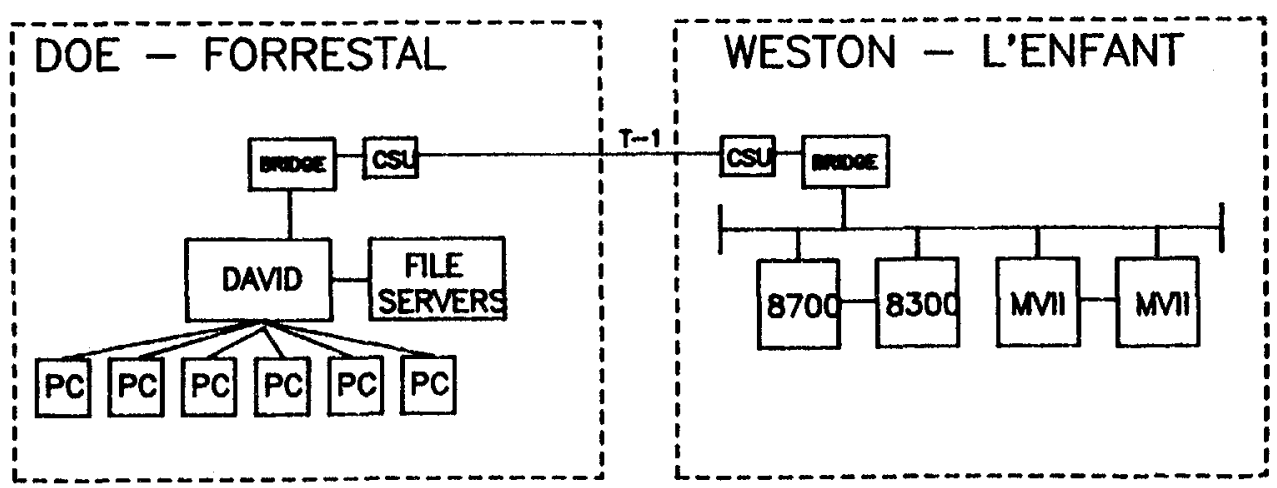




\section{Figure 3 OCRWM Information Systems and Data Bases}

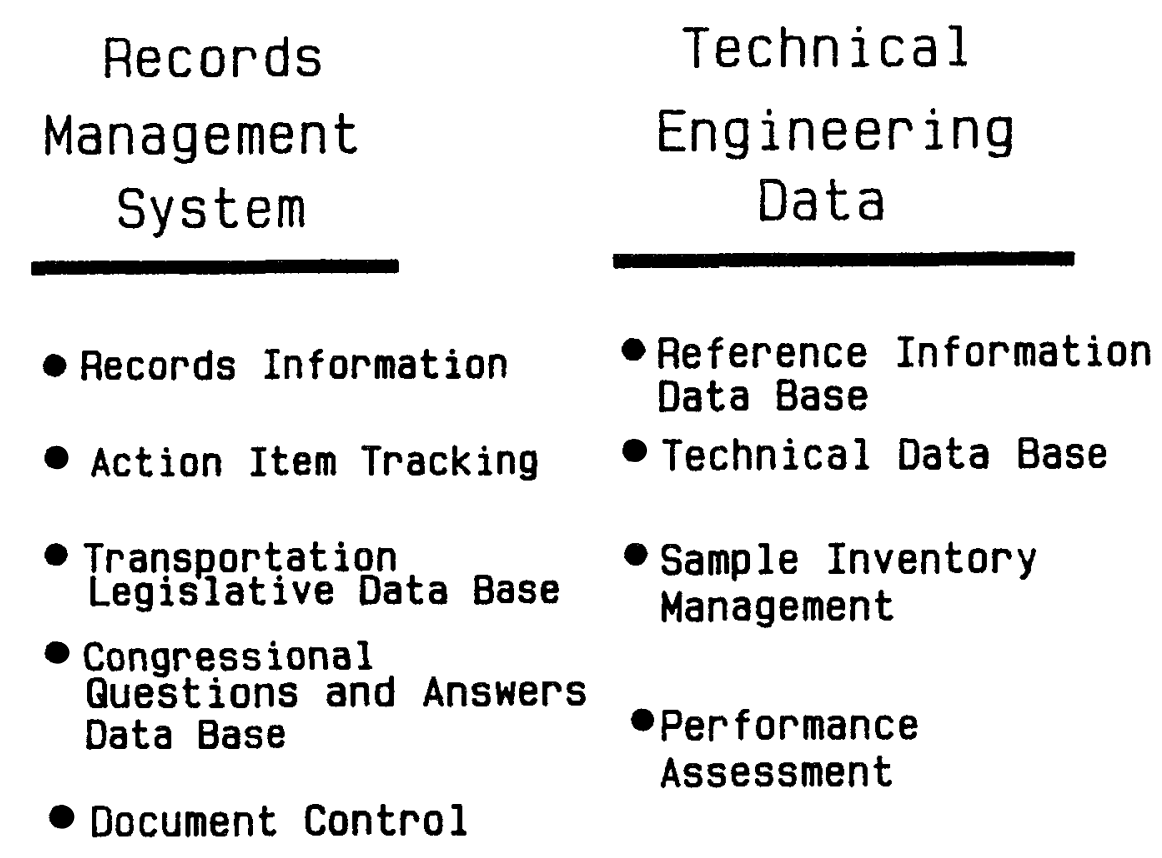

\section{Administrative System}

- Program Management

- Deliverables Tracking

- Budget Management
- Issues Tracking
- Commitment Tracking
- Document Review Tracking
- Comment Response Tracking
- Property Inventory Control




\subsubsection{RTTD/OWTD: Chicago, Illinois}

Office of Waste Technology Development (OWTD)/Willowbrook operates a VAXstation 3500 and a MicroVAX II. OWTD currently has installed a four-port Translan Bridge between OWTD/Willowbrook VAX 8700 and the Battelle-Columbus computer center. Repository Technology and Transportation Division (RTTD) is in the process of installing a MicroVAX II installed in the RTTD offices. Communications between OWTD and RTTD are currently under development.

\subsubsection{Idaho Falls, Idaho and Oak Ridge, Tennessee}

Both Idaho Falls, Idaho, and Oak Ridge, Tennessee provide information to RTTD-Chicago via dial-up commercial services. Idaho Falls data concern cask development for transportation, while Oak Ridge data concern the analysis of alternative transportation routes. Although both locations have functional local area telecommunications systems under the DOE Nationwide Telecommunications Services (DOENTS; see Section 5.1), neither currently has the functional wide area capability needed for the cost-effective high-speed telecommunications envisioned under Phase I. Oak Ridge is scheduled to be the Eastern Area Control Center for DOENTS and is also scheduled for connectivity with Germantown, Maryland (CSTM) in July of 1989. 


\subsection{INTRODUCTION: THE NEED FOR AN INTEGRATED} TELECOMMUNICATIONS NETWORK

In order to successfully implement the Nuclear Waste Policy Act (i.e., to site the Nation's first permanent geologic repository for spent fuel and high-level radioactive waste) OCRWM must, among other things, be equipped to readily produce, file, store, access, retrieve, and transfer a wide variety of technical and institutional data and information. The data and information regularly produced by members of the OCRWM Program supports, and will continue to support, a wide range of program activities. Some of the more important of these information communication-related activities include: responding to requests for information from parties affected by and/or interested in the program; (b) providing evidence of compliance with all relevant Federal, State, local, and Indian Tribe regulations, statutes, and/or treaties; and (c) supporting the preparation, submittal, and review of a license application to the NRC to authorize the construction of a geologic repository. With regard to these and other communications-related activities, there are two types of end-users to be supported: first, the technical user who will perform scientific/modeling computing and utilize high speed output printers and plotters: second, the administrative user who will perform data base searches, document production, file transfer, electronic messaging and All-In-1 (VAX-based) functions.

All users will need electronic mail, document and file transfer, and the capability of exchanging technical information electronically between parties involved in different aspects of the civilian radioactive waste management program. In order to meet this need, the telecommunications network must be carefully planned and implemented. In addition, periodic network performance assessments will prove critical in ensuring that the network continues to respond to OCRWM's information needs. For example, as additional local area networks (LANs) are linked to the OCRWM network they must have proper gateways and bridges, data throughputs must be maintained at acceptable levels; and network security, backup, and disaster recovery mechanisms must be revised to include them.

\subsubsection{Problems with the Existing Telecommunications Environment}

Because all program information and technical data are not centrally located, it is crucial that each of the sites have timely access to information located elsewhere. Otherwise, duplicate systems may be developed--if they do not already exist--and the probability of compromising the integrity of data and information stored and presented will be heightened.

Timely access to the data and information needed to perform program-related activities, regardless of where those data are located, is a critical prerequisite for ensuring that information management fully supports the construction and operation of a geologic repository. The telecommunications environment; consisting of OCRWM-HQ, YMPO/SAIC/M\&O, and RTTD/OWTD, is not presently able to routinely support off-site (remote) access to program data and information. As stated earlier, each site maintains its 
own battery of information systems and communications networks, including on-site LANs and extended LANs, which are not readily accessible across sites (i.e., in a wide area network configuration).

\subsubsection{Goals and Objectives of the New OCRWM Telecommunications Network}

The primary objectives of the new telecommunications network are: (a) to provide OCRWM Headquarters and the Project Offices with ready access to program data and information (see Figure 3) by introducing wide area networking technologies into the current OCRWM telecommunications environment; and, (b) to support the development, operation, and maintenance of the LSS. The LSS support will be guaranteed by incorporating known telecommunications requirements of the LSS into the functional requirements of the Phase I network.

The remainder of this section sets forth hardware and software requirements that must be met to facilitate development of a telecommunications network fully capable of supporting data and information flows across Phase I sites. The implementation of these requirements also provides OCRWM with a network base or foundation for the LSS.

GENERAL NETWORK REQUIREMENTS AND SPECIFICATIONS

\subsubsection{Conceptual Models and Designs}

Figure 4 illustrates the conceptual design for the proposed Phase I OCRWM wide area telecommunications network. Please refer to Appendices $A$ through $C$ for a brief review of LAN and WAN concepts (e.g., gateways, bridges) as well as for the implications of OCRWM's selection of local and wide area networking technologies.

\subsubsection{Phase I Telecommunications Network: General Functional Requirements}

Phase I will facilitate data base inquiries, document transfer, and electronic messaging. No voice carrier will be on the network in Phase I. Since OCRWM-HQ has directed the Project Offices to utilize DEC VAX, DM, and All-In-1, a DECnet VAX telecommunications environment is the most compatible with the OCRWM-HQ directive.

The ETHERNET LANs at each site will be connected to a remote bridge(s). The remote bridge(s) will provide the data link layer protocol required for connection of the localized (i.e., within, or on-site) LANs across sites. Because bridges will be used to connect each site, bandwidth usage must be closely examined to ensure that enough is allocated to each LAN in the network. If needed in Phase I, the introduction of digital T1 circuits, and the alternate routing and channel allocation capability of T1 multiplexors, would allow site-to-site bandwidth assignments to be more responsive (and adaptable) to changing data line requirements. 


\subsubsection{Operating in a DEC Environment: Node Addresses}

The Phase I hosts on the network will be primarily DEC VAXes, and the network will, in all likelihood, be DECnet (this is assumed pending CSTM's technical implementation plan). The nodes will, for the most part, be connected via ETHERNET LANs (DOE and GOSIP X802.3 standard; see Appendix 1) with the geographically dispersed ETHERNETs connected via remote bridges and high-speed communication lines.

The use of DECnet presents a unique set of problems because of the DECnet scheme for node addresses. Under "Phase IV DECnet," node addresses are composed of an area address between 1 and 63, and a node address between 1 and 1024 within that area. This scheme would seem to be adequate as it allows for up to 65,000 distinct node addresses. However, as organizations connect their existing DECnets to other organizations' DECnets, the potential for conflict in area numbers becomes very great. This is particularly true for the DOE, which may connect to a large number of DECnets at other organizations and laboratories, each of which may connect to a number of other DECnets that already have unique DECnet area numbers. If such were the case, there is a high probability that two organizations would have the same area numbers.

There are ways of working around the potential conflicts in network addressing, but these solutions almost always involve some level of compromise regarding which services are available between which nodes. For these reasons, it is absolutely essential that the DOE DECnet node numbers and parameters be centrally managed. This central management includes: (a) the assignment of node numbers, including area numbers; (b) whether each node is a level 1 router, a level 2 router, or an end node; (c) which node on an ETHERNET is the designated router; (d) the value of the "router priority" parameter for each node; and (e) which services are available between which nodes.

\subsubsection{Network Management, Control, and Maintenance}

With communications equipment and circuits located throughout the network, a single location should be designated as the control center. The control center's function would be to monitor all circuits on the network (including tail circuits along with the backbone), and to maintain the day-to-day operations of the network. The control center would also establish alternate routes, allocate bandwidth, and conduct performance monitoring of the network.

\subsubsection{Security}

The same general security measures presented in the OCRWM Office Automation Plan (November 1988) should be reflected in the OCRWM telecommunications network. To counter potential security risks, the following safeguards may be necessary depending on the nature and sensitivity of the information to be processed:

Multiple-level security and access control (including controlled access to storage devices at other sites). 


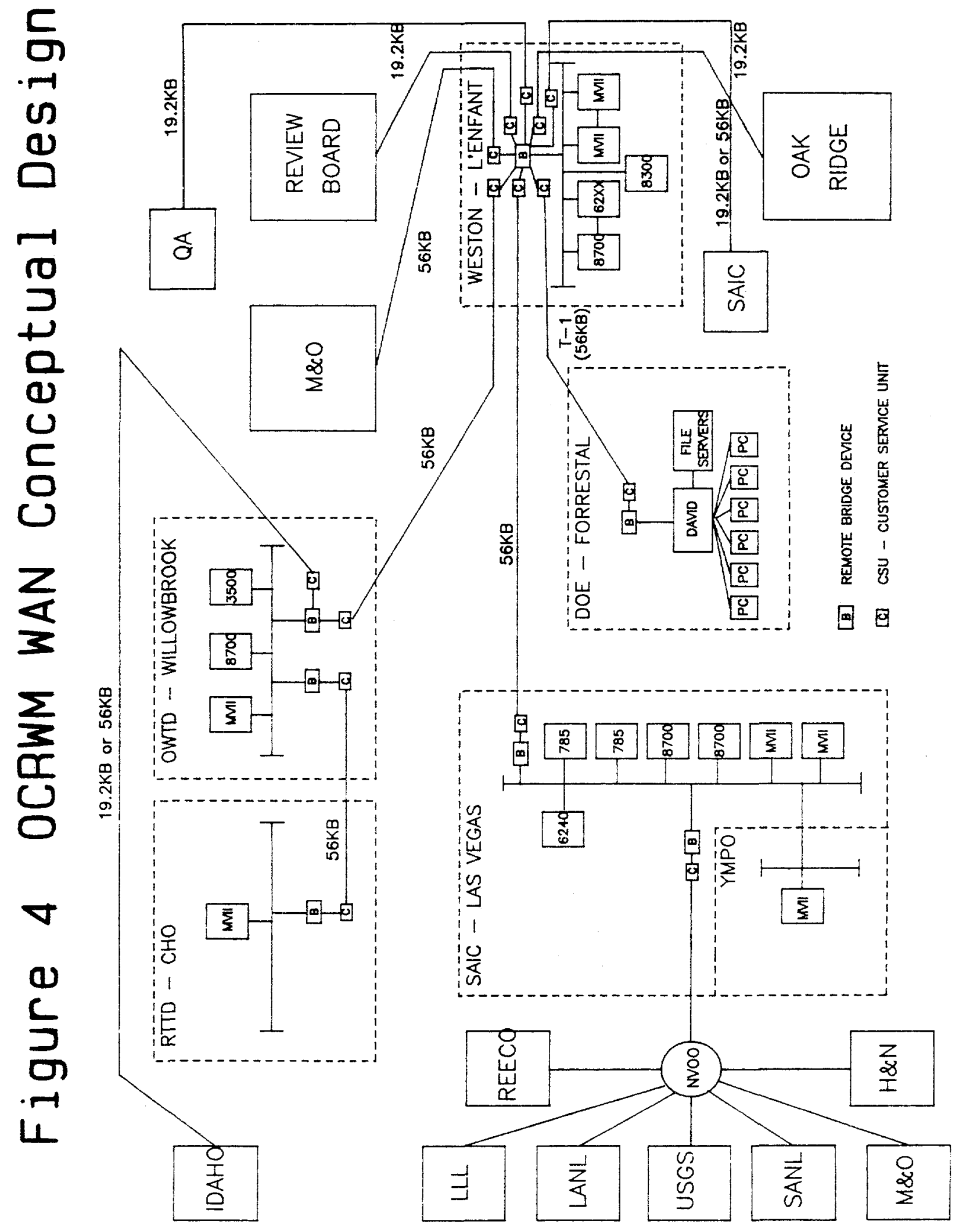


Failure of one node cannot bring down the whole network (node isolation star topology).

Audit trails.

Terminal controls.

Magnetic media controls.

Standardization.

Backup mechanism and offsite storage.

Intruder detection.

In addition to the preceding security measures, carrier (i.e., hardware) redundancy, network disaster-recovery, and uninterruptable power supply mechanisms must be established to reduce network downtime. Appendix D presents an overview of selected carrier redundancy alternatives. Finally, all DOE Orders governing the design, development, implementation, and operation of a telecommunications network must be considered in all stages of network design and introduction.

\section{SITE REQUIREMENTS}

This section will highlight site-specific hardware requirements for the proposed Phase I telecommunications network. A brief overview of a selected number of node-to-node communication alternatives (e.g., bandwidth/data line alternatives) is presented in Appendix E. These alternatives include: (a) T1 digital service; (b) DS0 channels; (c) 56 KBPS Digital Data Service (DDS); and (d) 9.6 to $19.2 \mathrm{KBPS}$ leased circuits. The site-specific hardware requirements are illustrated in Figure 4, OCRWM WAN - Phase I Conceptual Design. Figure 4 also includes the Nevada Operations Office (NVOO), Reynolds Electrical and Engineering Co. (REECO), the U.S. Geological Survey (USGS), SAIC/Las Vegas, the Nevada M\&O Contractor (M\&O), Holmes and Narver $(H \& N) / N e v a d a$, and the following National Laboratories: Lawrence Livermore National Laboratory (LLL), Los Alamos National Laboratory (LANL), and Sandia National Laboratory (SANL).

\subsubsection{Washington D.C.}

The OCRWM VAX cluster is located off-site and can be made accessible to OCRWM's Project Offices and other parties via a variety of communication service links running ETHERNET--which provides network communication services for VAX, as well as microcomputer, local and wide area networks. In order for OCRWM Headquarters to communicate with its Washington D.C. based Contractors, the Nuclear Waste Review Board, OCRWM Project Offices, and DOE Operations Offices, the telecommunications network should be implemented as follows:

- A bridge, with a minimum of four remote ports, should be procured and installed at OCRWM-HQ and Weston-Washington, D.C.; and at the Nuclear 
Waste Review Board, the D.C. M\&O Contractor, SAIC/McLean, Virginia, and the D.C. Quality Assurance (QA) Contractor, as each is ready to come on line.

o In addition to the bridges, each Phase I site must also procure and install a DSU for each communications line:

- OCRWM Headquarters: one DSU

- Weston/Washington, D.C.: six DSUs

- Nuclear Waste Review Board: one DSU

- D.C. M\&O Contractor: one DSU

- SAIC/McLean, Virginia: one DSU

- D.C. QA Contractor: one DSU

- A $56 \mathrm{~KB}$ circuit should be procured and installed between Weston/Washington, D.C. and each of the following sites: SAIC/Las Vegas (prototype under CSTM evaluation); OWTD/Willowbrook; and, the D.C. $\mathrm{M} \& \mathrm{O}$ Contractor (when it is ready to come on line).

o A 19.2 baud data line should be procured and installed between Weston-Washington, D.C. and: the Nuclear Waste Review Board (when it is ready to come on line); SAIC/McLean, Virginia; and the D.C. QA Contractor.

\subsubsection{Nevada}

In order for parties in Nevada to communicate with Washington, D.C., the requirements outlined in Section 3.3.1 must be met. In addition, a bridge with a minimum of four . remote ports, and two DSUs (one for each end), should be procured and installed in SAIC/Las Vegas (note: SAIC/Las Vegas should also be installing a local area network to facilitate on-site, as well as off-site access to their information resources). The document entitled "NNWSI Network Design" outlines the Las Vegas Network configuration designed to support communications between the Nevada Project Office and Nevada-based contractors.

\subsubsection{Chicago}

OWTD/Willowbrook has installed a four port TransLAN Bridge between Willowbrook and Columbus to provide access to the Battelle Columbus computer center and the Office of Transportation Systems and Planning/Columbus MicroVAX II. Recently, a one port TransLAN Bridge was installed at RTTD to provide access between RTTD and OWTD/Willowbrook. 


\section{RELATIONSHIP BETWEEN THE PROPOSED TELECOMMUNICATIONS NETWORK AND THE OCRWM LICENSING SUPPORT SYSTEM}

\subsection{THE LICENSING SUPPORT SYSTEM: PURPOSES AND OBJECTIVES}

The Licensing Support System (LSS) will be used to capture, store, access, and present (output) all records, regulations and tracking information that is deemed relevant to obtaining construction authorization for a geologic repository for the disposal of spent nuclear fuel and high-level radioactive waste as authorized by the Nuclear Waste Policy Act of 1982, and the Nuclear Waste Policy Amendments Act of 1987.

The Licensing Support System Preliminary Needs Analysis (DOE, 1989c) estimates that the LSS will need to support access to the LSS from multiple sites, in over a dozen cities throughout the U.S. Figure 5 illustrates the anticipated LSS National Communication Topology; that is, the sites OCRWM-HQ currently envisions to be part of the LSS telecommunications network.

The following is a breakdown of sites that are expected to participate in the proposed Phase II, LSS/OCRWM telecommunications network. The existing Phase I telecommunications sites will serve as regional communications "hubs" for participating LSS sites, as well as any other sites that may be brought on to the LSS/OCRWM network. In addition to the Phase I "hubs," San Antonio, Texas will be provided with direct access to the LSS host.

o Washington D.C.: OCRWM Headquarters and Contractors, Nuclear Waste Review Board, NRC (White Flint LAN).

o Nevada: DOE and Contractors for the YMPO Project located at the following sites - SAIC/Las Vegas, Nevada Operations Office, the Yucca Mountain Project Office, the University of Nevada at Las Vegas (all in Nevada); Los Alamos National Laboratory (Los Alamos, New Mexico), Lawrence Livermore National Laboratory (Livermore, California), United States Geologic Survey (Denver, Colorado), and Sandia National Laboratory (Albuquerque, New Mexico).

o Chicago, Illinois: DOE Operations Office and Contractors. RTTD/DOE Project staff and Contractors (except Battelle) and the Chicago Operations Office are co-located at Argonne National Laboratories. Battelle/OWTD is located 3 miles from Argonne in Willowbrook.

o San Antonio, Texas: Nuclear Regulatory Commission and Contractors.

The reader must bear in mind that as the LSS evolves and site-to-network access requirements are better understood, some sites may be removed from the network illustrated here, while others may be added. It should, however, be noted that while the number of sites included in the LSS telecommunications network will vary over time to 
Figure 5 Anticipated LSS Topology

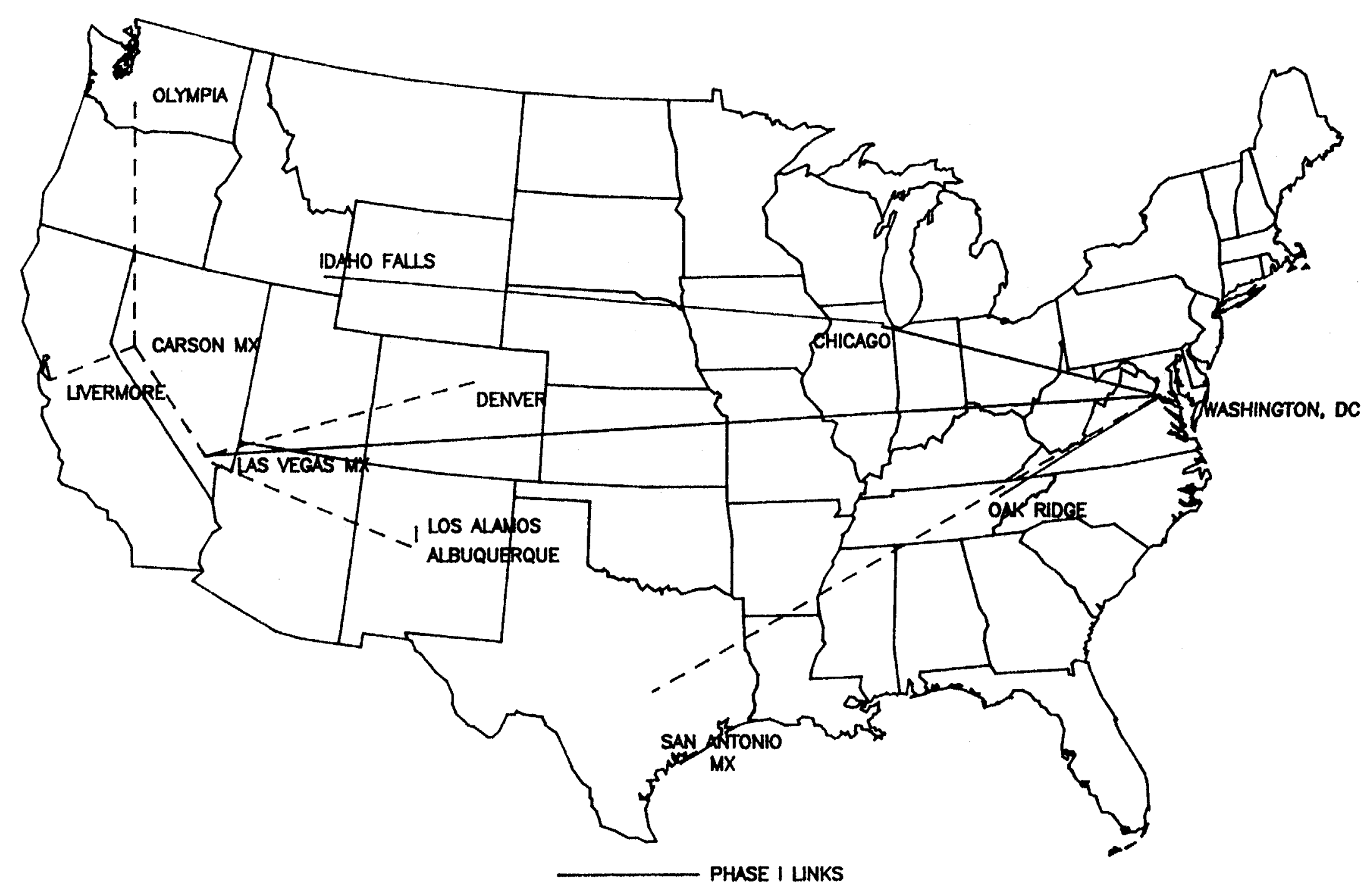

- - - - PHASE II LNKS 
reflect changing site-to-network access requirements, the majority (i.e., approximately 70 percent) of LSS workstations are expected to be in the Washington, D.C. and Las Vegas areas (see Licensing Support System Communications Conceptual Design; SAIC: August 12, 1988, Table 2, p. 5). This will have considerable implications for the overall requirements of the Phase II, LSS/OCRWM telecommunications network.

\section{2}

THE RELATIONSHIP BETWEEN THE PROPOSED TELECOMMUNICATIONS NETWORK AND THE LSS

As mentioned throughout this plan, the proposed Phase I telecommunications network will serve as the foundation for the Phase II, LSS/OCRWM telecommunications network. With this in mind, the Phase I network design must incorporate, to the extent practical, the functional and operating requirements of the Phase II, LSS/OCRWM network. The Phase I telecommunications network must readily accommodate the introduction of new sites; and, it must be able to support the transfer of a variety of types of LSS-related data and information (e.g., bit-mapped images) without OCRWM having to incur any significant expense on efforts to "upgrade" or modify the existing network. The requirements set forth in Section 3.3 reflect OCRWM's current telecommunications needs, and are believed to be consistent with the known requirements of the Phase II, LSS/OCRWM telecommunications network.

\section{LICENSING SUPPORT SYSTEM TELECOMMUNICATIONS REQUIREMENTS}

As stated in the introduction, this plan focuses primarily on Phase I of IRMD's efforts to implement an OCRWM-wide telecommunications network. This phase centers on establishing a viable telecommunications network across the program's Washington, D.C., Chicago, Illinois, and Las Vegas, Nevada sites. The specific operating requirements for Phase II of the OCRWM-wide telecommunications network (i.e. the LSS/OCRWM telecommunications network)--for which the Phase I network will serve as a foundation) will be addressed in a LSS communications plan to be issued later next year. The reader can refer to Figure 6 for a simplified view of the preliminary design of the LSS Communication Network. For a more extensive review of the proposed LSS Communication Network, please refer to the following DOE documents: 1987a, 1989a, 1989b, 1989c, and 1989d (see Reference Section of this plan). 
Figure 6 Preliminary Design LSS Communications Network

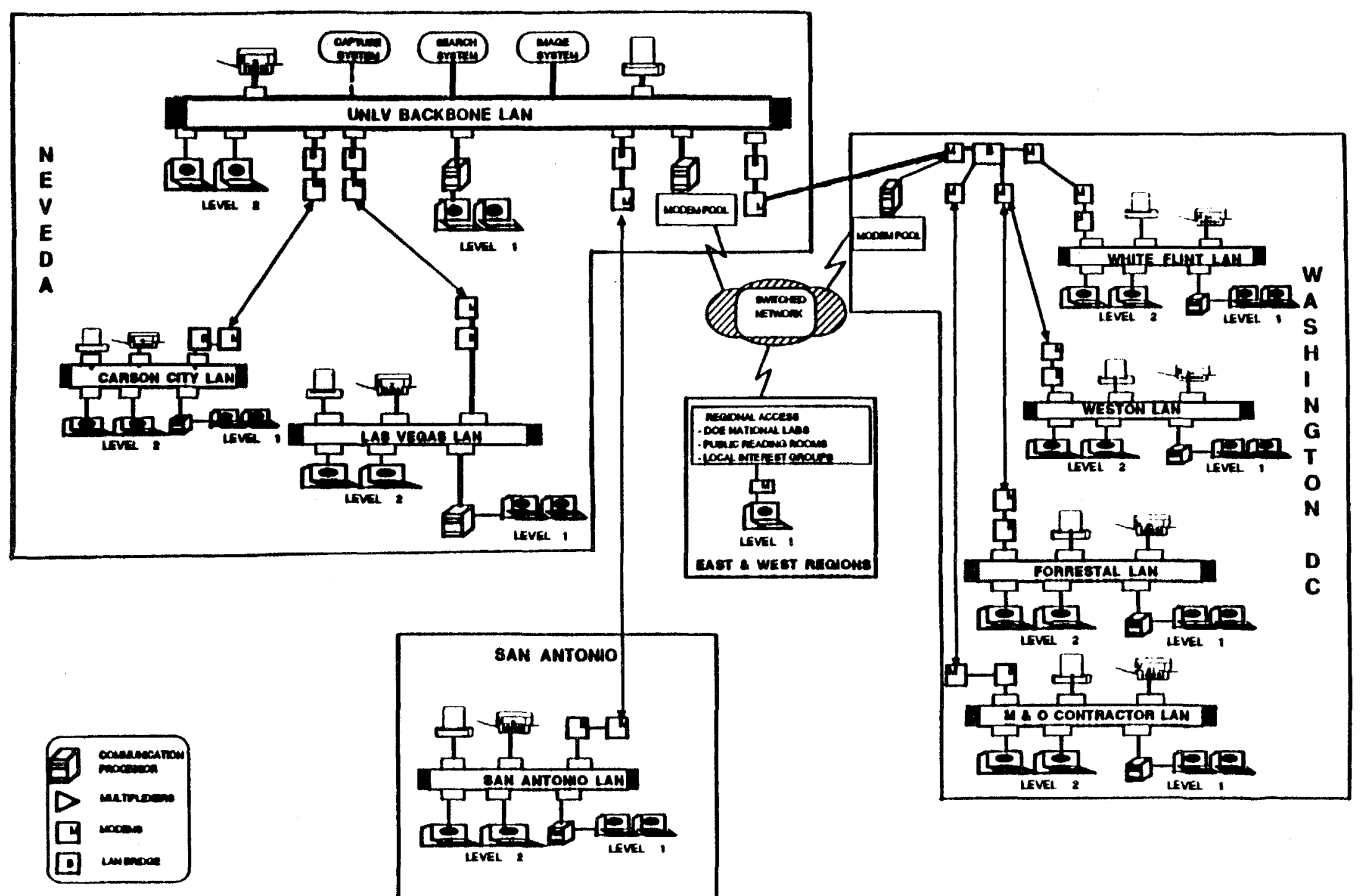


As with any effort within DOE to design, develop, implement, and maintain information resources, CSTM will play an integral role in ensuring that the desired objectives of such activities are achieved. OCRWM Headquarters will submit the functional requirements for the proposed Phase I and Phase II telecommunications networks detailed in this Plan to CSTM as soon as they are finalized. OCRWM Headquarters will then work with CSTM to develop the necessary technical implementation plans and procedures.

In addition to the purposes already stated in this plan, implementation of the proposed Phase I and Phase II wide area telecommunications networks is also intended, in part, to support and expand the DOE Nationwide Telecommunications Services (DOENTS), which already includes INTRANET and the OPMODEL. A brief description of each of these networks is provided below.

DOE Nationwide Telecommunications Services

A program was initiated in FY 1986 to consolidate/bundle nationwide circuits into wideband carrier systems in order to create an integrated service. These opportunities have come about through the introduction of low-cost fiber optics, as well as conventional copper links among major metropolitan areas.

The DOENTS is managed by DOE Headquarters; however, implementation will result in other DOE sites (e.g., OCRWM Program sites) either receiving or providing telecommunications support through use of its contractor systems, equipment, or services. The DOENTS, comprising the satellite backbone system, inter-city common-user trunks, and associated patch and test facilities, continues to evolve and expand to meet the emerging demand for telecommunications services in a cost-effective manner.

\section{INTRANET}

The INTRANET is a network that is funded by the Department of Energy in support of weapons research. It connects a number of National Laboratories via high-bandwidth circuits. Each node of this network consists of a Timeplex node capable of supporting multiple T1 circuits. These are multiplexing nodes that have the capability of breaking the primary bandwidth into many channels of lower bandwidths. There are monthly charges for INTRANET circuits based proportionately on the actual bandwidth utilized. The circuits between nodes consist of up to $6 \mathrm{~T} 1$ circuits per path. Within Nevada, the circuits are provided by a dedicated microwave system. Outside of Nevada, the circuits are leased from AT\&T and MCI. Redundant routing of circuits is provided to many of the node locations to assure continued network service in the event that a commercial circuit goes down. 
The INTRANET is maintained and managed by Reynolds Electrical and Engineering Co. (REECO) on behalf of the DOE. There is a complete Network Monitoring and Control Center (NCC) located at the central node site in Las Vegas. This center instantly detects problems with the commercial and microwave circuits and initiates procedures to restore network service. In addition, the NCC maintains contacts and works with the telecommunications staffs at the National Laboratories, where they also coordinate the diagnostics and correction of circuit problems.

\section{OPMODEL}

The Department has implemented a 15MBPS backbone nodal satellite telecommunications network (OPMODEL), with plans underway to upgrade to 60MBPS. The system was acquired to address the requirements of DOE locations nationwide for secure wide-band digital telecommunications services to accommodate large-scale transfers of engineering, administrative, and other information. While the primary driving forces behind the system were those associated with the expanding requirements of the weapons design and production facilities, the OPMODEL provides cost-effective transmission services for other DOE purposes.

\subsubsection{Phase I and Phase II Development and Implementation Schedules}

The development and implementation schedules for the proposed Phase I OCRWM wide area telecommunications network and the proposed Phase II LSS/OCRWM wide area telecommunications network are currently being developed in coordination with CSTM. These schedules will portray a multiphased approach to network development and implementation. The rationale behind "phasing" the introduction of telecommunications technologies into the OCRWM Program is quite simple. A phased approach is "scalable"; that is, it affords network designers and implementers the opportunity to adapt to changing functional requirements--to scale the network to the current and anticipated requirements of the network users. Phase I and Phase II implementation schedules will be included in subsequent revisions of this plan as they become available. 
The following documents were used to prepare this plan. The Information Resource Management Division at OCRWM would like to thank each of the authors for their contributions toward the development of the proposed telecommunications network.

Anderson, R.J., NNWSI Network Design (Battelle Columbus Division: Draft Report, September 30, 1988).

BPMD, 1988; OCRWM Telecommunications Network Plan: Phase I (Battelle Project Management Division: August 1988, Revision 1.0).

DOE, 1987a; Licensing Support System Functional Requirements and Design Concepts Report (Office of Resources Management, DOE Office of Civilian Radioactive Waste Management: March 31, 1987).

DOE, 1987b; Information Technology Resources Long-Range Plan: FY 1989-FY 1993 (DOE/MA-0310 December 1987).

DOE, 1989a; Licensing Support System Preliminary Data Scope Analysis (Office of Civilian Radioactive Waste Management: DOE/RW-0210 January 1989).

DOE, 1989b; Licensing Support System Conceptual Design Analysis (Office of Civilian Radioactive Waste Management: DOE/RW-0211 January 1989).

DOE, 1989c; Licensing Support System Preliminary Needs Analysis (Office of Civilian Radioactive Waste Management: DOE/RW-0212 January 1989).

DOE, 1989d; Licensing Support System; Benefit-Cost Analysis (Office of Civilian Radioactive Waste Management: DOE/RW-0213 January 1989).

DOE, 1989e; OCRWM Office Automation Plan (Office of Program Administration and Resources Management, Office of Civilian Radioactive Waste Management: Draft Report, January 1989).

SAIC, 1988; Licensing Support System Communications Conceptual Design (Science Applications International Corporation: August 12, 1988). 
OCRWM TELECOMMUNICATIONS NETWORK PLAN

A-1

REVISION 0.0: FEBRUARY 28, 1989

\title{
LOCAL AREA NETWORKS
}

\begin{abstract}
Appendix A
Reference: OCRWM Telecommunications Network Plan: Phase I (Battelle Project Management Division: August 1988, Revision 1.0).
\end{abstract}

Local Area Networks (LANs) provide a common backbone for connectivity of all devices. Dissimilar devices can use the LAN as a common point of entrance into the network. There are three standard LANs used in the computer industry, X802.3, X802.4, and X802.5. X802.3, ETHERNET, is the most prevalent. X802.4, MAP is a manufacturing-oriented LAN. X802.5, IBM's Token Ring LAN is probably the most widely used for PC LANs. Token Ring and MAP are limited in the number of physical nodes that can be placed on the network. ETHERNET on the other hand is a general purpose LAN that [when bridged] can connect thousands of nodes. Within OCRWM, limiting the potential number of nodes would [adversely] impact the exchange of information between parties; and as stated earlier, OCRWM-HQ has directed the Project Offices to utilize DEC VAX, DM and All-In-1. Thus, a DECnet VAX telecommunications environment is the most compatible with the OCRWM-HQ directive [and mission].

Connection to the network is achieved either through the use of terminal servers connected directly to the backbone or through PC communications cards connected at the board level on the PC and then through a Digital Multi-port Repeater.

Procurement and implementation of LANs will be the responsibility of each Project Office. Each Project Office must consider OCRWM's short and long-term telecommunications goals when selecting its software and hardware. For example, in order to exchange files and messages, gateways and bridges must be implemented properly. Omitting bridge and gateway considerations from LAN planning and procurement will jeopardize reaching OCRWM's telecommunications goals. Each Project Office will receive OCRWM-HQ approval prior to procurement of all LAN hardware and software to ensure OCRWM-wide compatibility. 


\section{EXTENDED LOCAL AREA NETWORKS}

\section{Appendix B}

Reference: OCRWM Telecommunications Network Plan: Phase I (Battelle Project Management Division: August 1988, Revision 1.0).

Extended Local Area Networks (LANs) connect two or more LANs into one large network. Devices on the network appear to the user as being on the same LAN. This has an advantage over multiplexors or other forms of communications in that devices can be easily moved from one LAN to another and still have the appearance of being on the same LAN. In a multiplexor or data switch environment, a multiplexor would have to be located in each building and then be interconnected to a central network processor. This adds additional cost in wiring and in telephone circuits to provide the service. In an extended LAN, however, bridges and gateways are used to interconnect the LANs into one large network. The bridges are the hardware used to forward data to interconnected LANs based on addressing information.

Each device on ETHERNET has a unique address that the bridge can use to determine whether packets are directed to a certain segment of the LAN. 


\title{
REMOTE EXTENDED LOCAL AREA NETWORKS
}

\begin{abstract}
Appendix C
Reference: OCRWM Telecommunications Network Plan: Phase I (Battelle Project Management Division: August 1988, Revision 1.0).
\end{abstract}

Extended LANs can be of two forms; a locally extended LAN and a remotely extended LAN. The locally extended LANs use higher-speed fiber optic or coaxial cable to interconnect the bridges between the LANs. Locally extended LANs are usually limited in distance. Most are implemented between two buildings or between floors of a building.

Remotely extended LANs, often referred to as Wide Area Networks (WANs), are usually geographically separated from each other over large distances. The connection of these LANs is usually limited by the bandwidth of the high speed circuit provided by carriers. Connections between the LANs can be done in two ways, either through a gateway or through a bridge.

A gateway differs from a bridge in that the gateway routes data based on the network layer protocols of the LAN. A bridge is specific to the network software being used. For instance, DECnet is the network software or transport layer used by the Digital Equipment Corporation. A bridge [using a DECnet router] will look at DECnet packets and determine the destination of the packet. Because a router is specific to the network layer, other network layer protocols cannot be routed through the same router. For instance, TCP/IP and XNS are two other network layer protocols. Data packets from devices that use these protocols could not be routed through Digital's DECnet router.

Remote ETHERNET bridges, on the other hand, route data packets based on the data link layer protocol of ETHERNET. Since the network layer protocols, DECnet, XNS, or TCP/IP all conform to ETHERNET X802.3, they all produce a similar packet. They all have an ETHERNET address for delivery of the packet.

Additionally, a data link layer bridge can route traffic much more quickly than a traditional router. A DECnet X.25 router can route traffic at the rate of 150-200 packets per second, while a data link layer bridge can route packets at a rate of 1500-2400 packets per second. This is an important consideration when total throughput must be considered.

An extended ETHERNET LAN is probably the most advantageous solution for the OCRWM telecommunications network. It provides a common backbone, it can be easily reconfigured to meet the demands placed on it by various users on the network, and other computing resources can be easily added at any point in the network. 


\section{SELECTED CARRIER REDUNDANCY ALTERNATIVES}

\section{Appendix D}

Reference: OCRWM Telecommunications Network Plan: Phase I (Battelle Project Management Division: August 1988, Revision 1.0).

If any of the circuits or hardware malfunction, communication will cease until the problem is resolved. A number of hardware redundancy alternatives are available to eliminate or significantly reduce the disruption of telecommunications service. With regard to providing carrier redundancy between Las Vegas, Nevada and Washington, D.C., cost-effective approaches are outlined below. [Similar] approaches also can be used to introduce carrier redundancy into the Chicago, Illinois site.

(1) A circuit between Las Vegas and another site (not Washington, D.C.) can be installed. Circuit routing must ensure that the circuit between the other site and Las Vegas, and the circuit between Washington, D.C. and Las Vegas are routed differently.

(2) An additional circuit can be installed between Las Vegas and Washington, D.C. Again, circuit routing must be handled to ensure that the two circuits are routed differently.

(3) Implement a "Switch 56." Switch 56 is not available in the Las Vegas area and is not planned at this time. AT\&T can provide the service, routing through Los Angeles or Phoenix; at the present time, however, this configuration is not cost-effective.

(4) Connect the Phase I telecommunications network to the DOE weapons network. OCRWM needs interactive communications. At this time, the DOE weapons network connecting the east and west coasts does not facilitate interactive communications. Interactive communications, however, is being explored by DOE for introduction into that network. 


\title{
BANDWIDTH AND DATA-LINE ALTERNATIVES
}

\author{
Appendix E
}

This Appendix provides a brief overview of selected bandwidth and data line (circuit) alternatives.

\section{(1) T1 Digital Service}

A T1 is an AT\&T designation for a digital communications path capable of handling 1.544 MBPS (million bits per second) two-way transmissions. Digital T1 service can be provided on various media including copper cable, digital microwave, fiber optical cable, and even satellite $\mathrm{KU}$ band channels.

T1 service has an advantage over traditional point-to-point analog or digital service in that cost for the T1 circuit is such that it can replace four or five 56 KBPS digital circuits, and yet provide additional bandwidth that otherwise could not be costjustified. The disadvantage of $\mathrm{T} 1$ service is that all carrier service is provided in twisted pair cable. If this circuit should go down, large amounts of data service and voice service could be adversely affected. Alternatives such as Switch T1, Accunet Reserve, etc. should be explored.

Another advantage to T1 service that must be considered is cost. Because the cost for a $\mathrm{T} 1$ circuit is relatively low in terms of bandwidth, special equipment can be used to take advantage of unused channels. For instance, one-half, or 12, of the channels could be used to provide data or voice service; while the other 12 channels could be used to provide alternative routing for another $\mathrm{T} 1$ circuit that is being used to service another organization in the network. Alternate routing is provided by T1 multiplexors placed at various points in the network. Not all Phase I or Phase II sites will require the capability to alternatively route voice and data channels from one point to another. Alternative routing using T1 mulitplexors has an advantage over traditional point-to-point carrier service. In a point-to-point environment, backup is not readily available without additional circuits. These circuits must be maintained in such a way as to provide alternative routing for the two different circuits. This could double the cost in most cases if sufficient backup is required.

T1 equipment is available for various types of applications from full alternate routing nodal T1 multiplexors to smaller T1 point-to-point multiplexors that just provide 24 $64 \mathrm{KBPS}$ channels. Each type of equipment must be evaluated to determine which is best suited for the location being served. 
(2) DS0 Channels

A DS0 channel is derivative of the T1 digital service. Commercial companies may now order only the portion of a T1 actually needed. DS0 channels, each a 64 KBPS channel, may be obtained one at a time up to an entire T1 (i.e., 24 DS0 channels). This arrangement allows the user to lease only the bandwidth needed for current data and voice transmission, while providing an easy migration to full $\mathrm{T} 1$ service as needs expand.

(3) 56 KBPS Digital Data Service

Since DS0 service is not currently available in some regions, the third type of link under consideration is the $56 \mathrm{KBPS}$ Digital Data Service (DDS). A 56 KBPS DDS is currently available from a number of vendors and provides enough capacity to extend full ETHERNET protocols to those nodes not now serviced with DS0 or T1 service. DDS is an extremely stable network of worldwide digital links.

(4) 9.6 to 19.2 KBPS Linked Circuits

The fourth type of network circuit includes simple 9.6 (9600 baud) to $19.2 \mathrm{KBPS}$ leased lines. 